\title{
SÍNDROME DE TERSON EN MALFORMACIÓN DE ARNOLD CHIARI I
}

\section{TERSON'S SYNDROME ASSOCIATED WITH ARNOLD-CHIARI I MALFORMATION}

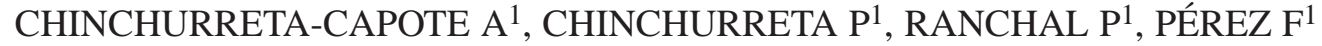

\begin{abstract}
RESUMEN
Caso clínico: Se presenta una paciente con Síndrome de Terson (ST) bilateral tras descompresión quirúrgica medular como tratamiento de su malformación de Arnold Chiari tipo I. Se estudia y compara la progresión de sus hemorragias prerretinianas tras tratamiento con láser Nd:YAG en su ojo derecho y observación en el izquierdo, formándose en éste una membrana epirretiniana.

Discusión: El tratamiento del ST se basa en observación y/o cirugía según ciertas pautas. La hialoidectomía YAG también consigue buenos resultados en casos seleccionados.

Palabras clave: Síndrome de Terson, hemorragia vítrea, hemorragia subhialoidea, Malformación de Arnold-Chiari, membrana epirretiniana.
\end{abstract}

\begin{abstract}
Case report: We present a patient with bilateral Terson's syndrome after brainstem surgical decompression as treatment for her Arnold-Chiari I malformation. We have studied and compared the progression of preretinal hemorrhage after Nd:YAG laser treatment of the right eye and observation of the left, and also the formation of an epiretinal membrane in the left eye.

Discussion: Treatment of Terson's syndrome is based on observation or surgery according to different criteria. Hyaloidectomy with YAG laser produces good results in selected cases (Arch Soc Esp Oftalmol 2007; 82: 113-116).
\end{abstract}

Key words: Terson syndrome, vitreous hemorrhage, subhyaloid hemorrhage, Arnold-Chiari Malformation, epiretinal membrane.

\section{INTRODUCCIÓN}

El Síndrome de Terson (ST) se define como una hemorragia vítrea seguida de cualquier tipo de sangrado intracraneal, debido a una hipertensión intra- craneal transmitida a través del espacio de la vaina del nervio óptico (NO) (1).

La malformación de Arnold-Chiari se caracteriza por un desplazamiento caudal de las estructuras de la fosa posterior a través del foramen magno.

Recibido: 12/5/06. Aceptado: 13/2/07.

Servicio de Oftalmología. Hospital Regional Universitario Carlos Haya. Málaga. España.

1 Licenciado en Medicina.

Correspondencia:

Ana Chinchurreta Capote

Hospital Civil C.H. Carlos Haya. Servicio de Oftalmología

Plaza del Hospital Civil, s/n

Málaga

España

E-mail: achinchu@hotmail.com 


\section{CASO CLÍNICO}

Mujer de 45 años que es remitida desde el servicio de neurocirugía por pérdida de visión súbita bilateral tras cirugía de derivación ventrículoperitoneal de un higroma subdural temporal y absceso occipital craneal. Todo ello resultado de una complicación postquirúrgica subaguda. Quince días antes había sido intervenida de descompresión preventiva medular por Malformación de Arnold-Chiari I (figs. 1 y 2).

La paciente contaba con una clínica de 16 años de evolución, con cefaleas de intensidad leve, inestabilidad, mareos rotatorios, vómitos autolimitados de duración variable y nistagmus vertical leve desde hacía un año. Éste último ayudó a la sospecha y diagnóstico de la malformación congénita, ya que el resto de su sintomatología la relacionaba con la colitis ulcerosa que sufría hacía años.

La agudeza visual (AV) en la primera exploración fue de movimiento de manos en ambos ojos.

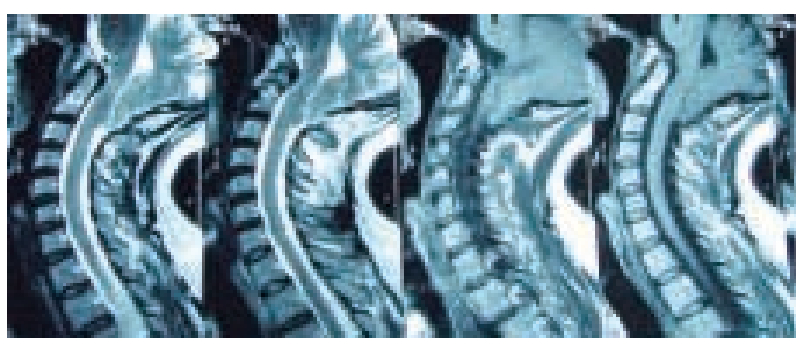

Fig. 1: RMN cráneovertebral sagital. Herniación tonsilar cerebelar a través del agujero magno de más de 5 mm hasta nivel de C2-C3. Malformación de ArnoldChiari I.

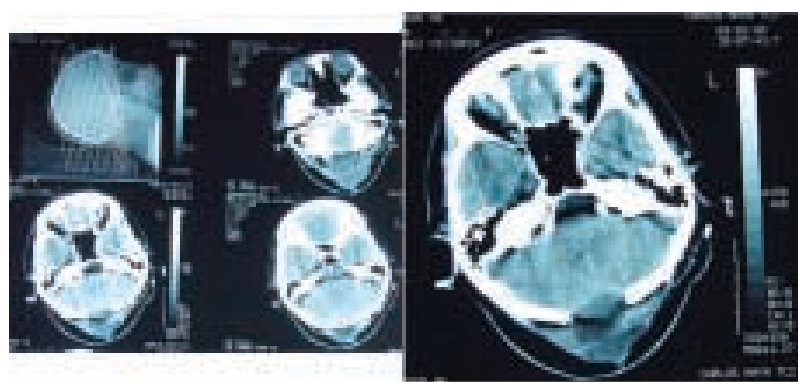

Fig. 2: a) TAC craneal con contraste. Imágenes tras cirugía de malformación de Arnold-Chiari. Craniectomía y laminectomía C1-C2. b) A mayor aumento, en corte cervical se observa colección líquida con pared engrosada que se realza con contraste y burbujas en su interior. Absceso occipital.
Las reacciones pupilares fueron de $2+$ sin defecto pupilar aferente relativo. Presentaba un nistagmus en sacudida hacia abajo de frecuencia alta y amplitud media. El fondo de ojo mostró hemorragia retrohialoidea premacular y vítrea bilateral, confirmada con tomografía óptica de coherencia (OCT) y ecografía ocular (figs. 3 y 4). Todos estos hechos fueron compatibles con ST.

Se le realizó una hialoidectomía posterior en su ojo derecho (OD) previa instilación de colirio anestésico y uso de láser Nd:YAG 15 pulsos (rango de 4-7 $\mathrm{mJ} /$ pulso) en la parte más inferior del hematoma. La hialoides posterior se rompió y drenó inmediatamente sangre a la cavidad vítrea (fig. 5). Se opta por una actitud expectante en su ojo izquierdo (OI).

$\mathrm{La} \mathrm{AV}$ al año, tras varias revisiones con notable mejoría, era de 0,6 en el OD y 0,1 en el OI. La OCT reveló una membrana epirretiniana premacular de reciente aparición en su OI (fig. 6). La frecuencia del nistagmus bajó y la amplitud pasó a ser leve. El

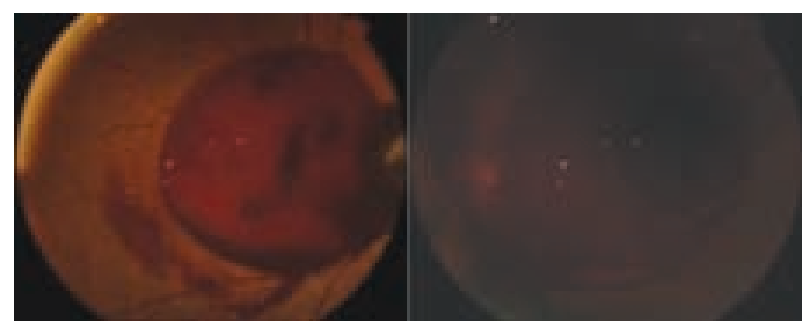

Fig. 3: Densa hemorragia prerretinal bilateral cubriendo mácula en paciente con Síndrome de Terson. En OI se observa además hemorragia vítrea que impide visualizar con claridad estructuras retinianas.

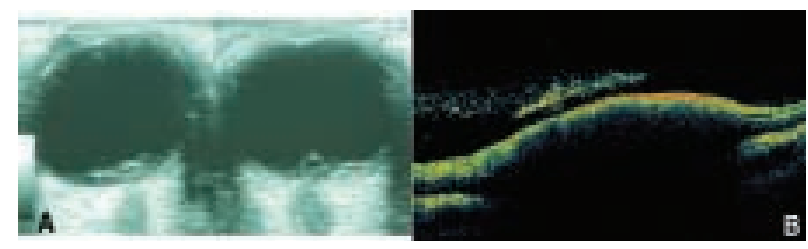

Fig. 4: a) Ecografía ocular bilateral en Síndrome de Terson. Sección vertical sobre el área macular. Se observa una membrana en forma de cúpula sobre el polo posterior ocular y separada de éste correspondiendo al hematoma prerretinal. b) Tomografía óptica de coherencia macular del mismo paciente. Línea hiperreflectante abombada debajo de la hialoides posterior, efecto sombra sobre la retina inferior debido al acúmulo de sangre retrohialoidea. Hiperreflectividad dispersa en vítreo premacular por hemorragia vítrea. 


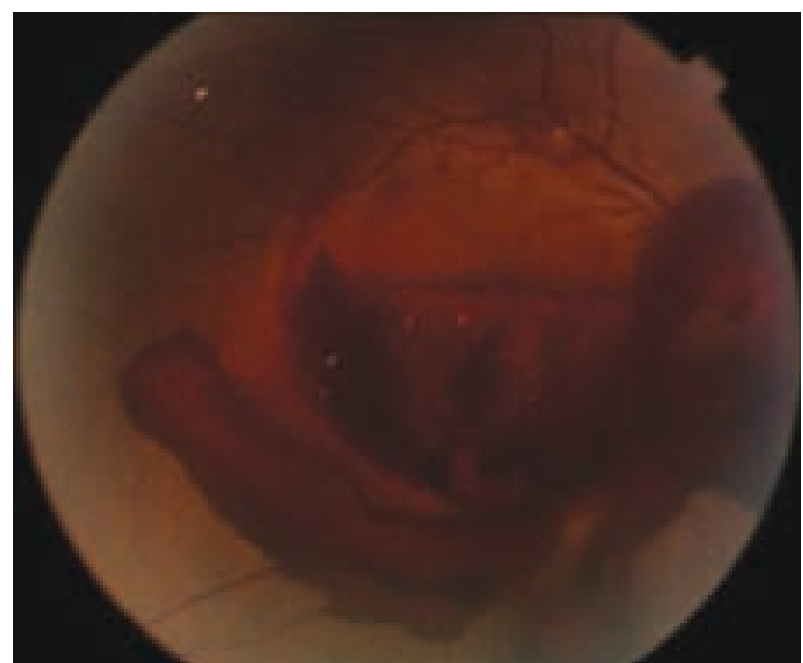

Fig. 5: Drenado de sangre a la cavidad vítrea en $O D$ inmediatamente después de la fotodisrupción con láser Nd: YAG. Nivel de sangre visible dentro el hematoma como consecuencia de la sedimentación de los hematies.

hemovítreo bilateral seguía en reabsorción de forma lenta y progresiva.

La enferma rechazó cirugía ocular alguna, estando satisfecha con el resultado visual alcanzado.

\section{DISCUSIÓN}

El tratamiento de la malformación de ArnoldChiari es quirúrgico, mediante la descompresión de la unión cráneovertebral (2).

Las complicaciones tempranas de ésta técnica son muy raras y entre ellas están la meningitis, el hematoma, la infección y el higroma subdural. Nuestra enferma sufrió a la vez estas dos últimas,

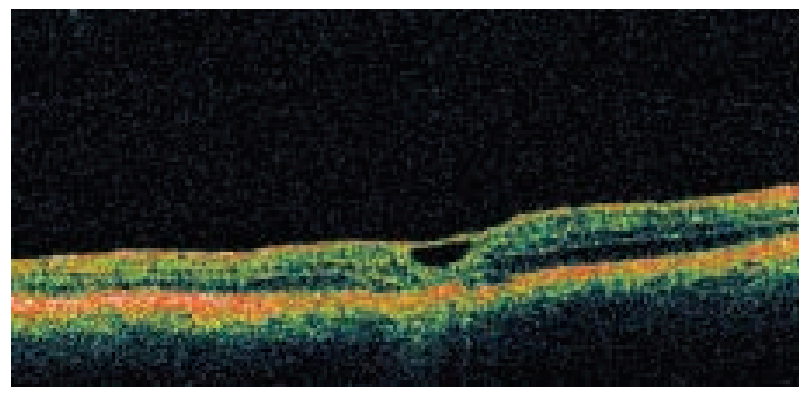

Fig. 6: Tomografía óptica de coherencia de membrana epirretiniana macular en OI de un paciente con Síndrome de Terson al año de observación. Membrana hiperreflectante que cubre completamente el contorno foveal. las cuales se resolvieron con técnicas de derivación. Se han descrito a su vez, complicaciones de las derivaciones lumbo y ventrículoperitoneales como son la hemorragia intracraneal tras el paso del catéter por el parénquima cerebral y la herniación tonsilar con hipertensión intracraneal (2). Este último hecho provocó en la paciente la citada hemorragia premacular.

En el ST, la hipertensión intracraneal se trasmite dentro de la vaina del NO; ésta se dilata, comprimiendo y obstruyendo a su vez las anastomosis retinocoroideas. Todo ello conlleva a una marcada reducción del drenaje venoso ocular con subsecuente hipertensión y hemorragia venosa retiniana (1).

Las opciones de tratamiento del ST incluyen observación, vitrectomía vía pars plana (3) o administración de láser Nd: YAG para drenar el hematoma a la cavidad vítrea (4). La indicación de cada una depende de las características del paciente.

La observación se puede considerar como primera indicación según los siguientes factores: unilateralidad, extensión de la opacidad vítrea, signos de reabsorción espontánea, edad, ocupación, necesidades visuales, estado psicológico y neurológico, papel familiar y riesgo de nueva cirugía.

La hemorragia vítrea en el ST se resuelve en un año muy lentamente y la media de mejoría visual oscila entre 20/300 y 20/30. El hemovítreo puede persistir incluso 6 años (3).

Las complicaciones de una hemorragia prerretinal persistente son: membrana epirretiniana (del 25 al $70 \%$ según distintos estudios), agujero retiniano, disrupción del epitelio pigmentario de la retina y efectos tóxicos retinianos de la hemoglobina (3).

Tres meses puede ser un periodo razonable para la observación y determinación del aclaramiento vítreo. A menos que esté contraindicado por las condiciones generales (como era nuestro caso) se debe realizar vitrectomía.

En casos seleccionados se puede utilizar láser $N d$ : YAG para la fotodisrupción del hematoma; conociendo sus posibles complicaciones, tanto el cirujano como el paciente. Entre ellas destacamos el agujero macular, el desprendimiento de retina y la formación de membrana epirretiniana (5).

En nuestra enferma, se adoptó en el OD ésta última opción de tratamiento, ya que era buena colaboradora y no necesitaría anestesia general. La terapia se llevó a cabo de forma segura y sin ninguna complicación posterior. No así ocurrió en su OI, el cual 
desarrolló una membrana epirretiniana al año de observación.

\section{BIBLIOGRAFÍA}

1. Ogawa T, Kitaoka T, Dake Y, Amemiya T. Terson syndrome: a case report suggesting the mechanism of vitreous hemorrhage. Ophthalmology 2001; 108: 1654-1656.

2. Steinmetz, MP, Benzel EC. Surgical management of Chiari malformation. Neurosurg $Q 2003$; 13: 105-112.
3. Kuhn F, Morris R, Witherspoon CD, Mester V. Terson syndrome. Results of vitrectomy and the significance of vitreous hemorrhage in patients with subarachnoid hemorrhage. Ophthalmology 1998; 105: 472-477.

4. Monshizadeh R, Tariq Bhatti M, Levine L, Tabandeh $H$. Photodisruption of dense preretinal hemorrhage with $\mathrm{Nd}$ : $Y A G$ in a child with Terson's syndrome. J AAPOS 2002; 6: $56-58$.

5. Kwok AK, Lai TY, Chan NR. Epiretinal membrane formation with internal limiting membrane wrinkling after $\mathrm{Nd}$ : $Y A G$ laser membranotomy in valsalva retinopathy. Am J Ophthalmol 2003; 136: 763-766. 